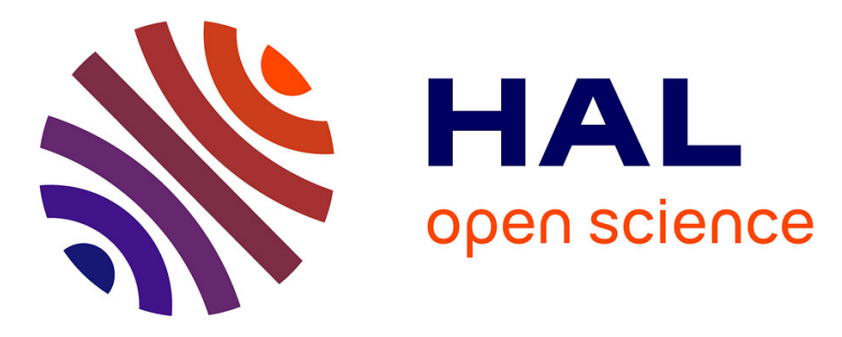

\title{
An Agent-Based Architecture for Personalized Recommendations
}

\author{
Amel Ben Othmane, Andrea G. B. Tettamanzi, Serena Villata, Nhan Le \\ Thanh, Michel Buffa
}

\section{- To cite this version:}

Amel Ben Othmane, Andrea G. B. Tettamanzi, Serena Villata, Nhan Le Thanh, Michel Buffa. An Agent-Based Architecture for Personalized Recommendations. H. Jaap van den Herik; Joaquim Filipe. Agents and Artificial Intelligence. ICAART 2016, 10162, Springer, pp.96 - 113, 2017, Lecture Notes in Computer Science, 10.1007/978-3-319-53354-4_6 . hal-01531141

\section{HAL Id: hal-01531141 \\ https://hal.science/hal-01531141}

Submitted on 1 Jun 2017

HAL is a multi-disciplinary open access archive for the deposit and dissemination of scientific research documents, whether they are published or not. The documents may come from teaching and research institutions in France or abroad, or from public or private research centers.
L'archive ouverte pluridisciplinaire HAL, est destinée au dépôt et à la diffusion de documents scientifiques de niveau recherche, publiés ou non, émanant des établissements d'enseignement et de recherche français ou étrangers, des laboratoires publics ou privés. 


\title{
An Agent-based Architecture for Personalized Recommendations
}

\author{
Amel Ben Othmane ${ }^{1}$, Andrea Tettamanzi ${ }^{3}$, Serena Villata ${ }^{2}$, \\ Nhan LE Thanh ${ }^{3}$, and Michel Buffa ${ }^{3}$ \\ 1 WIMMICS Research Team, INRIA, I3S, UMR 7271 \\ Sophia Antipolis, France, \\ amel.ben-othmane@inria.fr \\ 2 CNRS, I3S, UMR 7271 \\ Sophia Antipolis, France \\ villata@i3s.unice.fr \\ 3 Univ. Nice Sophia Antipolis, I3S, UMR 7271 \\ Sophia Antipolis, France \\ \{andrea.tettamanzi, nhan.le-thanh, michel.buffa\}@unice.fr
}

\begin{abstract}
This paper proposes a design framework for a personalized multi-agent recommender system. More precisely, the proposed framework is a multi-context based recommender system that takes into account user preferences to generate a plan satisfying those preferences. Agents in this framework have a Belief-Desire-Intention (BDI) component based on the well-known BDI architecture. These BDI agents are empowered with cognitive capabilities in order to interact with others agents. They are also able to adapt to the environment changes and to the information coming from other agents. The architecture includes also a planning module based on ontologies in order to represent and reason about plans and intentions. The applicability of the proposed model is shown through a simulation in the NetLogo environment.
\end{abstract}

\section{Introduction and motivation}

Human activities take place in particular locations at specific times. The increasing use of wearable devices enables the collection of information about these activities from an heterogeneous set of actors varying in physical, cultural, and socioeconomic characteristics. Generally, the places you have spent regularly or occasionally time in, reflect your lifestyle, which is strongly associated to your socioeconomic features. This amount of information about people, their relations, and their activities are valuable elements to personalize healthcare being sensitive to medical, social, and personal characteristics of individuals. Besides this, the decision-making process in human beings is based not only on logical objective elements, but also emotional ones that are typically extra-logical. As a result, the behavior can also be explained by other approaches, which additionally consider emotions, intentions, beliefs, motives, cultural and social constraints, impulsive actions, and even the simple willingness to try. Hence, 
building recommender systems that take user behavior into account requires a step toward personalization.

To the best of our knowledge, there are no recommender systems that combine all these features at the same time. Consider the following motivating example that had driven this research: Bob, a 40 year-old adult, wants to get back to a regular physical activity $(p a)$. Bob believes that a regular physical activity reduces the risk of developing a non-insulin dependant diabetes mellitus $(r d)$. Mechanisms that are responsible for this are weight reduction $(w r)$, increased insulin sensitivity, and improved glucose metabolism. Due to his busy schedule $(b s)$, Bob is available on weekends ( $a v)$ only. Hence, he would be happy if he can do his exercises only on weekends $(w)$. Bob prefers also not to change his eating habits $(e h)$. Besides all the aforementioned preferences, Bob should take into account his medical concerns and refer to a healthcare provider for monitoring. This scenario exposes the following problem: how can we help Bob to select the best plan to achieve his goal based on his current preferences and restrictions? This problem raises different challenges. First, the proposed solution should take into account Bob's preferences and restrictions (e.g., medical and physical concerns) in the recommendation process. Second, information about the environment in which Bob acts and people that might be in relationship with him may have impact in his decision-making process. Third, the system should be able to keep a trace of Bob's activities in order to adapt the recommendation according to his progress. Finally, the information or data about Bob's activities is distributed geographically and temporarily.

In order to address these challenges, multi-agent systems stand as a promising way to understand, manage and use distributed, large-scale, dynamic, and heterogeneous information. The idea is to develop recommender systems able to help users confronted with situations in which they have too many options to choose from, with the aim of assisting them to explore and filter out their preferences from a number of different possibilities. Based on this real-world application scenario, we propose in this paper a multi-agent-based recommender system where agents are described using the BDI model as a multi-context system. The system's goal is to recommend a list of activities according to the user preferences. We propose also an extension of the BDI model to deal with the social dimension and the uncertainty in dynamic environments.

The originality of what we propose with respect to existing works is the combination of an extended possibilistic BDI approach with multi-context systems. The resulting framework is then used as a healthcare recommender system. There are several advantages deriving from such combination. First, the use of a multi-context architecture allows us to have different syntaxes, e.g., the ontology to represent and reason about plans and intentions. Besides this, we believe that extending the classical BDI model with goals and social contexts better reflects human behavior. Moreover, the proposed approach deals with goal-belief consistency, and defines a belief revision process. However, the idea of extending the BDI model with social contexts is not novel: different works explored trust 
or reputation $[1,2]$. In our approach, we consider trust measures between two agents only if they are similar.

The reminder of this paper is organized as follows: Section 2 includes a literature overview about the related work. In Section 3, we summarize the main concepts on which this work is based. We introduce after, in Section 4, the multi-context BDI agent framework. In order to show how the model works, we describe in Section 5 a real-world scenario in healthcare domain, and we describe its simulation in Section 6. Conclusions end the paper.

\section{Related Work}

Research in agent-based recommender systems is increasing in order to address the challenges raised by a growing number of real-world applications. For a taxonomy of recommender agents on the Internet, we refer the reader to [3]. Several works propose to use a cognitive architecture as a base for a recommender system. Next, we will focus on works using the well known BDI architecture.

Casali et al. proposed in [4] a Travel Assistant agent that helps a tourist to choose holiday packages. They used a graded BDI agent model based on multi-context systems to deal with information uncertainty and graded notions of beliefs, desires and intentions, and a modal many-valued logic approach for modeling agents. An implementation of the proposed model is later presented in [5] and [6]. Results concluded that BDI agents are useful to build recommender systems. Nevertheless, as pointed in [7], this approach needs further research to adapt the agent behavior in a dynamic environment.

In [8], the authors propose a framework for personalized recommendations in e-commerce. They use the cognitive architecture as a middle layer between the user, and a set of recommerders instead of using it as a recommender. However, the proposed framework still in a preliminary stage and needs further improvements, e.g., to enable the communication with the user.

Another example of a multi-agent recommender system using a BDI architecture is studied in [9]. The proposed system, SHOpping Multi-Agent System (SHOMAS), aims at helping users to identify a shopping or leisure plan. The architecture of the user agent is based on both Case-Based Reasoning and the Beliefs Desires Intentions architectures (CBR-BDI). The combination of the two architectures allows dynamic re-planning in a dynamic environment.

In [10], an Interest Based Recommender System (IBRS) is proposed for the personalization of recommendations. The IBRS is an agent-based recommender system that takes into account users' preferences to generate personalized recommendations. Agents are based on the BDI architecture empowered with cognitive capabilities and interact with other users using argumentation. A travel case study is used to experiment the model.

Our work takes a different approach compared to the aforementioned approaches. It is based on a full-fledged possibilistic approach and includes a revision process for beliefs and intentions . 


\section{Background}

In this section, we summarize the main insights on which the present contribution is based.

An agent in a BDI architecture is defined by its beliefs, desires and intentions. Beliefs encode the agent's understanding of the environment, desires are those states of affairs that an agent would like to accomplish and intentions those desires that the agent has chosen to act upon. Many approaches tried to formalize such mental attitudes (e.g., [11], [12], [13] and [14]). However, all these works concentrated on the human decision-making process as a single approach without considering social influences. They did not take the gradual nature of beliefs, desires, and intentions into account. Incorporating uncertainty and different degrees of attitudes will help the agent in the decision-making process. In order to represent and reason about uncertainty and graded notions of beliefs, desires, and intentions, we follow the approach proposed in [15], where uncertainty reasoning is dealt with by possibility theory. Possibility theory is an uncertainty theory dedicated to handle incomplete information. It was introduced by [16] as an extension to fuzzy sets which are sets that have degrees of membership in $[0,1]$. Possibility theory differs from probability theory by the use of dual set functions (possibility and necessity measures) instead of only one. A possibility distribution assigns to each element $\omega$ in a set $\Omega$ of interpretations a degree of possibility $\pi(\omega) \in[0,1]$ of being the right description of a state of affairs. It represents a flexible restriction on what is the actual state with the following conventions:

$-\pi(\omega)=0$ means that state $\omega$ is rejected as impossible;

$-\pi(\omega)=1$ means that state $\omega$ is totally possible (plausible).

While we chose to adopt a possibilistic BDI model to include gradual mental attitudes, unlike [15], we use multi-context systems (MCS) [17] to represent our BDI agents. According to this approach, a BDI model is defined as a group of interconnected units $\left\{C_{i}\right\}, i \in I, \Delta_{b r}$, where:

- For each $i \in I, C_{i}=\left\langle L_{i}, A_{i}, \Delta_{i}\right\rangle$ is an axiomatic formal system where $L_{i}, A_{i}$ and $\Delta_{i}$ are the language, axioms, and inference rules respectively. They define the logic for context $C_{i}$ whose basic behavior is constrained by the axioms.

$-\Delta_{b r}$ is a set of bridge rules, i.e., rules of inference which relate formulas in different units.

The way we use these components to model BDI agents is to have separate units for belief $B$, desires $D$ and intentions $I$, each with their own logic. The theories in each unit encode the beliefs, desires, and intentions of specific agents and the bridge rules $\left(\Delta_{b r}\right)$ encode the relationships between beliefs, desires and intentions. We also have two functional units $C$ and $P$, which handle communication among agents and allow to choose plans that satisfy users desires. To summarize, using the multi-context approach, a BDI model is defined as follows: 


$$
A g=\left(\{B C, D C, I C, P C, C C\}, \Delta_{b r}\right)
$$

where $B C, D C, I C$ represent respectively the Belief Context, the Desire Context and the Intention Context. $P C$ and $C C$ are two functional contexts corresponding to Planning and Communication Contexts.

The use of MCS offers several advantages when modeling agent architectures: it gives a neat modular way of defining agents, which allows from a software perspective to support modular architectures and encapsulation.

\section{The Multi-context BDI Framework}

The BDI agent architecture we are proposing in this paper extends Rao and Georgeffs well-known BDI architecture [12]. We define a BDI agent as a multicontext system being inspired by the work of [17]. Following this approach, our BDI agent model, visualized in Figure 1, is defined as follows:

$$
A g=\left(\{B C, D C, G C, S C, P C, I C, C C\}, \Delta_{b r}\right)
$$

where $G C$ and $S C$ represent the Goal and the Social Contexts, respectively.

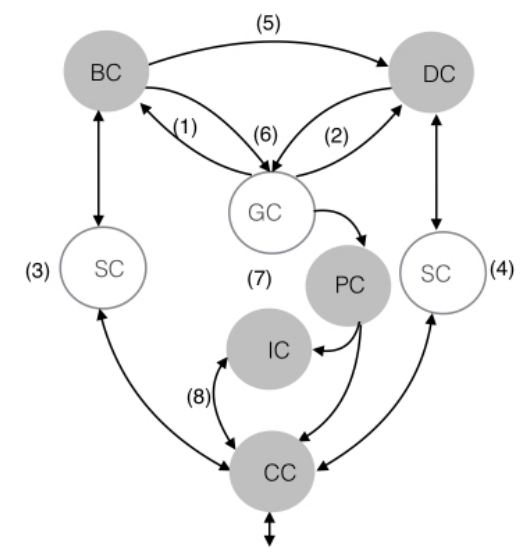

Fig. 1: The extended Multi-context BDI agent model.

In order to reason about beliefs, desires, goals and social contexts we follow the approach developed by da Costa Pereira and Tettamanzi $[15,18]$ where they adopt a classical propositional language for representation and possibility theory to deal with uncertainty.

Let $\mathcal{A}$ be a finite set of atomic propositions, and $\mathcal{L}$ be the propositional language such that $\mathcal{A} \cup\{\top, \perp\} \subseteq \mathcal{L}$ and $\forall \phi, \psi \in \mathcal{L}, \neg \phi \in \mathcal{L}, \phi \vee \psi \in \mathcal{L}, \phi \wedge \psi \in \mathcal{L}$. These propositions can contain temporal elements that are left as future work. As in [15], $\mathcal{L}$ is extended, and we will denote with $\Omega=\{0,1\}^{\mathcal{A}}$ the set of all 
interpretations on $\mathcal{A}$. An interpretation $\omega \in \Omega$ is a function $\omega: \mathcal{A} \rightarrow\{0,1\}$ assigning a truth value $p^{\omega}$ to every atomic proposition $p \in \mathcal{A}$ and, by extension, a truth value $\phi^{\omega}$ to all formula $\phi \in \mathcal{L}$. $[\phi]$ denotes the set of all interpretations satisfying $\phi$. (i.e., $[\phi]=\{\omega \in \Omega: \omega \vDash \phi\}$ ).

In the planning and intentions contexts, we propose an ontological representation for plans and intentions to provide the agents with a computer-interpretable description of the services they offer, and the information they have access to (workout plans in our case). In the following subsections, we will outline the different theories defined for each context in order to complete the specification of our multi-context agent model.

\subsection{Belief Context}

The BC language and semantics In order to represent beliefs, we use the classical propositional language with additional connectives, following [15]. We introduce also a fuzzy operator $B$ over this logic to represent agents beliefs. The belief of an agent is then represented as a possibility distribution $\pi$. A possibility distribution $\pi$ can represent a complete preorder on the set of possible interpretations $\omega \in \Omega$. This is the reason why, intuitively, at a semantic level, a possibility distribution can represent the available knowledge (or beliefs) of an agent. When representing knowledge, $\pi(\omega)$ acts as a restriction on possible interpretations and represents the degree of compatibility of the interpretation $\omega$ with the available knowledge about the real world. $\pi(\omega)=1$ means that is totally possible for $\omega$ to be the real world. As in [15], a graded belief is regarded as a necessity degree induced by a normalized possibility distribution $\pi$ on the possible worlds $\omega$. The degree to which an agent believes that a formula $\Phi$ is true is given by:

$$
B(\phi)=N([\phi])=1-\max _{\omega \leftleftarrows \phi}\{\pi(\omega)\}
$$

An agent's belief can change over time because new information arrives from the environment or from other agents. A belief change operator is proposed in [15], which allows to update the possibility distribution $\pi$ according to new trusted information. This possibility distribution $\pi^{\prime}$, which induces the new belief set $B^{\prime}$ after receiving information $\phi$, is computed from the possibility distribution $\pi$ with respect to the previous belief set $B\left(B^{\prime}=B * \frac{\tau}{\Phi}, \pi^{\prime}=\pi * \frac{\tau}{\Phi}\right)$ as follows: for all interpretations $\omega$,

$$
\pi^{\prime}(\omega)= \begin{cases}\frac{\pi(\omega)}{\Pi(\{\phi\})} & \text { if } \omega \vDash \phi \text { and } B(\neg \phi)<1 ; \\ 1 & \text { if } \omega \vDash \phi \text { and } B(\neg \phi)=1 ; \\ \min \{\pi(\omega),(1-\tau)\} & \text { if } \omega \not \models \phi .\end{cases}
$$

where $\tau$ is the trust degree towards a source about an incoming information $\phi$.

BC Axioms and Rules Belief context axioms include all axioms from classical propositional logic with weight 1 as in [19]. Since a belief is defined as a necessity measure, all the properties of necessity measures are applicable in this context. 
Hence, the belief modality in our approach is taken to satisfy these properties that can be regarded as axioms. The following axiom is then added to the belief unit:

$$
B C: B(\phi)>0 \rightarrow B(\neg \phi)=0
$$

It is a straightforward consequence of the properties of possibility and necessity measures, meaning that if an agent believes $\phi$ to a certain degree then it cannot believe $\neg \phi$ at all. Other consequences are:

$$
\begin{aligned}
& B(\phi \wedge \psi) \equiv \min \{B(\phi), B(\psi)\} \\
& B(\phi \vee \psi) \geq \max \{B(\phi), B(\psi)\}
\end{aligned}
$$

The inference rules are:

$-B(\neg p \vee q) \geq \alpha, B(p) \geq \beta \vdash B(q) \geq \min (\alpha, \beta)$ (modus ponens)

$-\beta \leq \alpha, B(p) \geq \alpha \vdash B(p) \geq \beta$ (weight weakening)

where $\vdash$ denotes the syntactic inference of possibilistic logic.

\subsection{Desire Context}

Desires represent a BDI agent's motivational state regardless its perception of the environment. Desires may not always be consistent. For example, an agent may desire to be healthy, but also to smoke; the two desires may lead to a contradiction. Furthermore, an agent may have unrealizable desires; that is, desires that conflict with what it believes possible.

The DC language and semantics In this context, we make a difference between desires and goals. Desires are used to generate a list of coherent goals regardless to the agent's perception of the environment and its beliefs. Inspired from [18], the language of $D C\left(L_{D C}\right)$ is defined as an extension of a classical propositional language. We define a fuzzy operator $D^{+}$, which is associated with a satisfaction degree $\left(D^{+}(\phi)\right.$ means that the agent positively desires $\left.\phi\right)$ in contrast with a negative desire, which reflects what is rejected as unsatisfactory. For sake of simplicity, we will only consider the positive side of desires in this work, and the introduction of negative desires is left as future work.

In this theory, da Costa Pereira and Tettamanzi [15] use possibility measures to express the degree of positive desires. Let $u(\omega)$ be a possibility distribution called also qualitative utility (e.g., $u(\omega)=1$ means that $\omega$ is fully satisfactory). Given a qualitative utility assignment $u$ (formally, a possibility distribution), the degree to which the agent desires $\phi \in L_{D C}$ is given by:

$$
D(\phi)=\Delta([\phi])=\min _{\omega \vDash \phi}\{u(\omega)\}
$$

where $\Delta$ is a guaranteed possibility measure that, given a possibility distribution $\pi$, is defined as follows:

$$
\Delta(\Omega)=\min _{\omega \in \Omega}\{\pi(\Omega)\}
$$


DC Axioms and Rules The axioms consist of all properties of possibility measures such as $D(\phi \vee \psi) \equiv \min \{D(\phi), D(\psi)\}$. The basic inference rules, in the propositional case, associated with $\Delta$ are:

- $[D(\neg p \wedge q) \geq \alpha],[D(p \wedge r) \geq \beta] \vdash[D(q \wedge r) \geq \min (\alpha, \beta)]$ (resolution rule)

- if $p$ entails $q$ classically, $[D(p) \geq \alpha] \vdash[D(q) \geq \alpha]$ (formula weakening)

- for $\beta \leq \alpha,[D(p) \geq \alpha] \vdash[D(p) \geq \beta]$ (weight weakening)

- $[D(p) \geq \alpha] ;[D(p) \geq \beta] \vdash[D(p) \geq \max (\alpha, \beta)]$ (weight fusion).

\subsection{Goal Context}

Goals are sets of desires that, besides being logically "consistent", are also maximally desirable, i.e., maximally justified. Even though an agent may choose some of its goals among its desires, nonetheless there may be desires that are not necessarily goals. The desires that are also goals represent those states of the world that the agent might be expected to bring about precisely because they reflect what the agent wishes to achieve. In this case, the agent's selection of goals among its desires is constrained by three conditions. First, since goals must be consistent and desires may be inconsistent, only the subsets of consistent desires can be the potential candidates for being promoted to goal-status, and also the selected subsets of consistent desires must be consistent with each other. Second, since desires may be unrealizable whereas goals must be consistent with beliefs (justified desires), only a set of feasible (and consistent) desires can be potentially transformed into goals. Third, desires that might be potential candidates to be goals should be desired at least to a degree $\alpha$. Then, only the most desirable, consistent, and possible desires can be elected as goals.

The $G C$ language and semantics The language $L_{G C}$ to represent the Goal Context is defined over the propositional language $L$ extended by a fuzzy operator $G$ having the same syntactic restrictions as $D^{+} . G(\phi)$ means that the agent has goal $\phi$. As explained above, goals are a subset of consistent and possible desires. Desires are adopted as goals because they are justified and achievable. A desire is justified because the world is in a particular state that warrants its adoption. For example, one might desire to go for a walk because he believes it is a sunny day and may drop that desire if it starts raining. A desire is achievable, on the other hand, if the agent has a plan that allows it to achieve that desire.

GC Axioms and Rules Unlike desires, goals should be consistent, meaning that they can be expressed by the $D_{G}$ axiom ( $D$ from the KD45 axioms [12]) as follows:

$$
D_{G} \quad G C: G(\phi)>0 \rightarrow G(\neg \phi)=0
$$

Furthermore, since goals are a set of desires, we use the same axioms and deduction rules as in $D C$. Goals-beliefs and goals-desires consistency will be expressed with bridge rules as we will discuss later on in the paper. 


\subsection{Social Context}

One of the benefits of the BDI model is to consider the mental attitude in the decision-making process, which makes it more realistic than a purely logical model. However, this architecture overlooks an important factor that influences this attitude, namely the sociality of an agent. There are a number of ways in which agents can influence each other mental states, e.g., authority where an agent may be influenced by another to adopt a mental attitude whenever the latter has the power to guide the behavior of the former, trust where an agent may be influenced by another to adopt a mental attitude merely on the strength of its confidence in the latter, or persuasion where an agent may be influenced to adopt another agent mental state via a process of argumentation or negotiation. In this work, we will only consider trust as a way by which agents can influence each others.

The $S C$ language and semantics In our model, we consider a multi-agent system MAS consisting of a set of $N$ agents $\left\{a_{1}, . ., a_{i}, . . a_{N}\right\}$. The idea is that these agents are connected in a social network such as agents with the same goal. Each agent has links to a number of other agents (neighbors) that change over time. In this paper, we do not consider dynamic changes in the social network, but we assume to deal with the network in a specific time instant. Between neighbors, we assume a trust relationship holds. The trustworthiness of an agent $a_{i}$ towards an agent $a_{j}$ about an information $\phi$ is interpreted as a necessity measure $\tau \in[0,1]$, as in [20], and is expressed by the following equation:

$$
T_{a_{i}, a_{j}}(\phi)=\tau
$$

where $a_{i}, a_{j} \in M A S=\left\{a_{1}, . ., a_{i}, . ., a_{N}\right\}$. Trust is transitive in our model, which means that, trust does not hold only between agents having a direct link to each other, but indirect links are also considered. Namely if agent $a_{i}$ trusts agent $a_{k}$ to a degree $\tau_{1}$, and $a_{k}$ trusts agent $a_{j}$ with a trust degree $\tau_{2}$ then $a_{i}$ can infer its trust for agent $a_{j}$, and $T_{a_{i}, a_{j}}(\phi)=\min \left\{\tau_{1}, \tau_{2}\right\}$.

$\boldsymbol{S C}$ Axioms and Rules As sociality is expressed as a trust measure, which is interpreted as a necessity measure, $S C$ axioms include properties of necessity measures as in $B C$ (e.g., $N(\phi \wedge \psi) \equiv \min \{N(\phi), N(\psi)\})$.

When an agent is socially influenced to change its mental attitude, by adopting a set of beliefs and/or desires, the latter should maintain a degree of consistency. Those rules will be expressed with bridge rules that link the social context to the belief and the desire contexts.

\subsection{Planning and Intention Contexts}

The aim of this functional context is to extend the BDI architecture in order to represent plans available to agents and provide a way to reason over them. 
In this context, we are inspired by [21] to represent and reason about plans and intentions. Plans are described using ontologies. [22] defines an ontology as 'the specification of conceptualizations used to help programs and humans to share knowledge'. According to the World Wide Web Consortium ${ }^{4}$ (W3C), ontologies or vocabularies define the concepts and relationships used to describe and represent an area of concern. We use the $5 \mathrm{~W}^{5}$ (Who, What, Where, When, Why) vocabulary which is relevant for describing different concepts and constraints in our scenario. The main concepts and relationships of this ontology are illustrated in Figure 2.

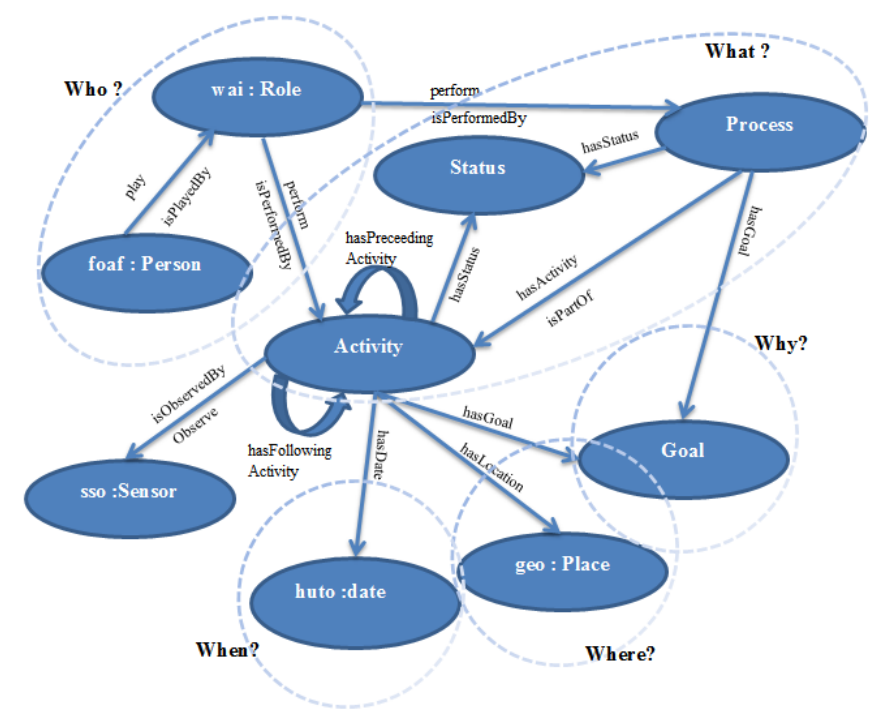

Fig. 2: The main concepts and relationships of the $5 \mathrm{~W}$ ontology.

The main task of this context is to select plans that satisfy maximally the agents goals. To go from the abstract notions of desires and beliefs to the more concrete concepts of goals and plans, as illustrated in Figure 3, the following steps are considered: (1) new information arrives and updates beliefs or/and desires which trigger goals update; (2) these goal changes invoke the Plan Library. The selection process is expressed by Algorithm 1 which looks in a knowledge base (KB) for all plans that satisfy maximally these goals; $C B$ and/or $C F$ techniques can be used in the selection process but will be investigated more thoroughly in further work. The algorithm complexity is significantly reduced since we discard from the beginning goals without plans; (3) one or more of these plans is then chosen and moved to the intention structure; and (4) a task (intention) is selected

\footnotetext{
${ }^{4}$ http://www.w3.org/standards/semanticweb/ontology

${ }^{5}$ http://ns.inria.fr/huto/5w/
} 


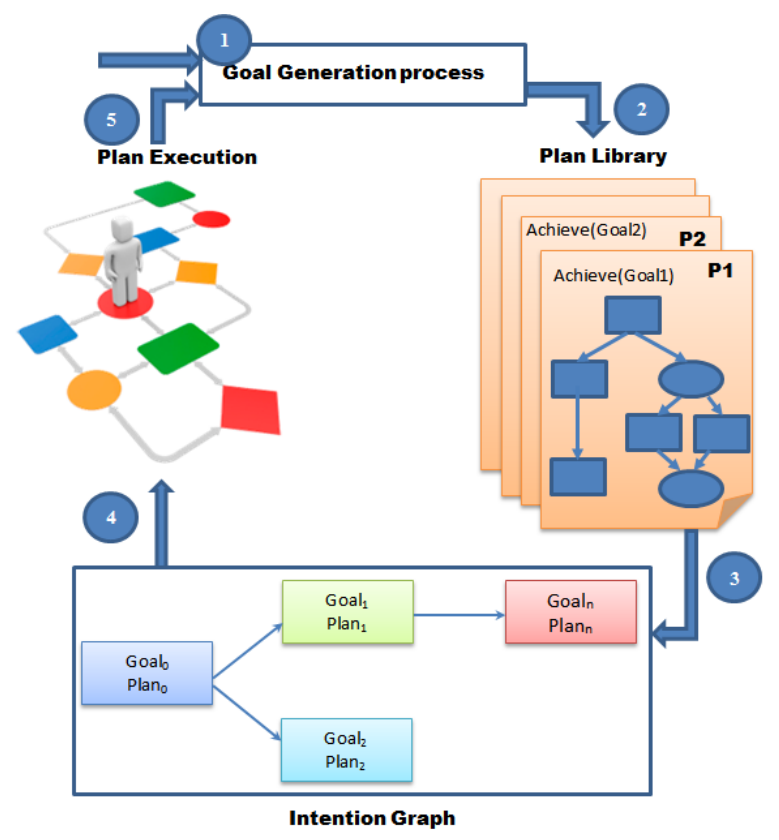

Fig. 3: Planning and Intention Contexts

for execution and once executed or failed this leads to the update of the agents beliefs (5).

\subsection{Bridge Rules}

There are a number of relationships between contexts that are captured by socalled bridge rules. A bridge rule is of the form:

$$
u 1: \phi, u 2: \psi \rightarrow u 3: \theta
$$

and it can be read as: if the formula $\phi$ can be deduced in context $u 1$, and $\psi$ in $u 2$, then the formula $\theta$ has to be added to the theory of context $u 3$. A bridge rule allows to relate formulae in one context to those in another one. In this section, we present the most relevant rules, illustrated by numbers in Figure 1. For all the agents in the MAS, the first rule relating goals to beliefs can be expressed as follows:

$$
(1) \vDash G C: G\left(a_{i}, \phi\right)>0 \rightarrow B C: B\left(a_{i}, \neg \phi\right)=0
$$

which means that if agent $a_{i}$ adopts a goal $\phi$ with a satisfaction degree equal to $\beta_{\phi}$ then $\phi$ is believed possible to a degree $\beta_{\phi}$ by $a_{i}$. Concerning rule (2) relating the goal context to the desire context, if $\phi$ is adopted as goal then it is positively desired with the same satisfaction degree.

$$
(2) \vDash G C: G\left(a_{i}, \phi\right)=\delta_{\phi} \rightarrow D C: D^{+}\left(a_{i}, \phi\right)=\delta_{\phi}
$$




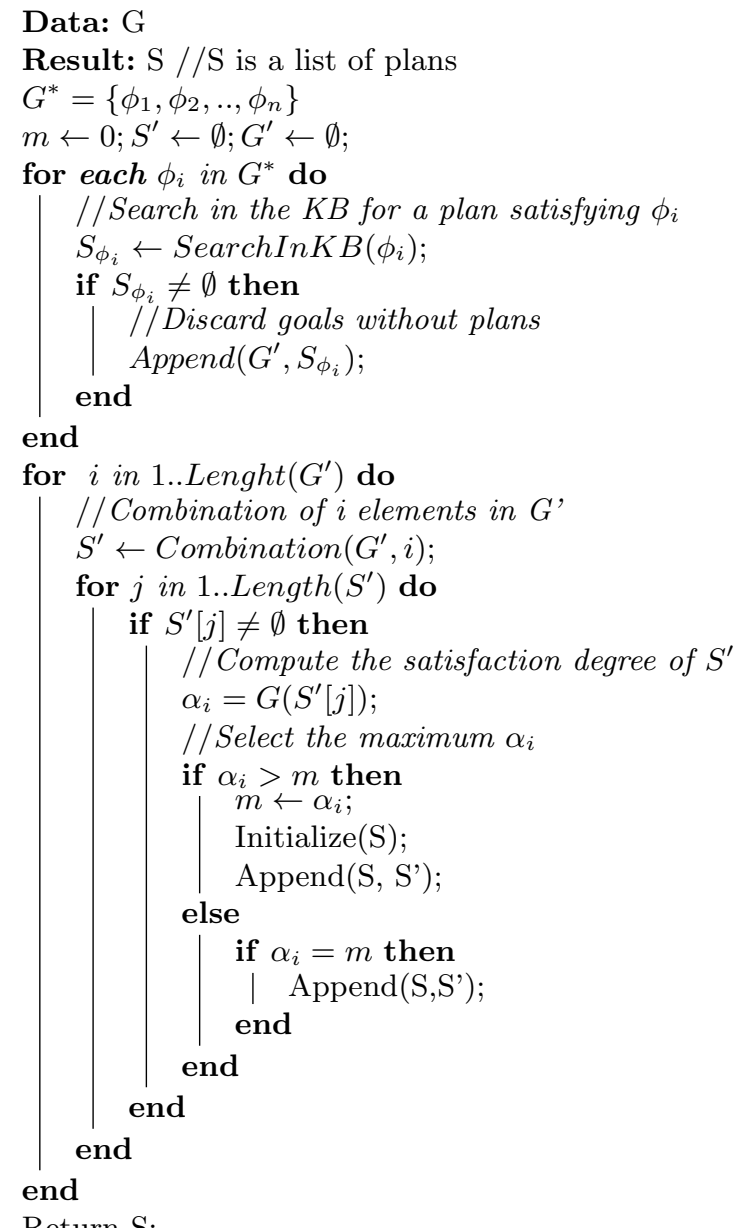

Algorithm 1: RequestForPlan Function

An agent may be influenced to adopt new beliefs or desires. Beliefs coming from other agents are not necessarily consistent with the agent's individual beliefs. This can be expressed by the following rule:

$$
(3) \vDash B C: B\left(a_{j}, \phi\right)=\beta_{\phi}, S C: T_{a_{i}, a_{j}}(\phi)=t \rightarrow B C: B\left(a_{i}, \phi\right)=\beta_{\phi}^{\prime}
$$

where $\beta_{\phi}^{\prime}$ is calculated using Equation 1 with $\tau=\min \left\{\beta_{\phi}, t\right\}$ to compute the possibility distribution, and Equation 1 to deduce the Belief degree.

Similarly to beliefs, desires coming from other agents need not to be consistent with the agent's individual desires. For example, an agent may be influenced by another agent to adopt the desire to smoke, and at the same time having the desire to be healthy, as shown by the following rule:

$$
(4) \vDash D C: D^{+}\left(a_{j}, \psi\right)=\delta_{\psi}, S C: T_{a_{i}, a_{j}}(\psi)=\tau \rightarrow D C: D^{+}\left(a_{i}, \psi\right)=\delta_{\psi}^{\prime}
$$




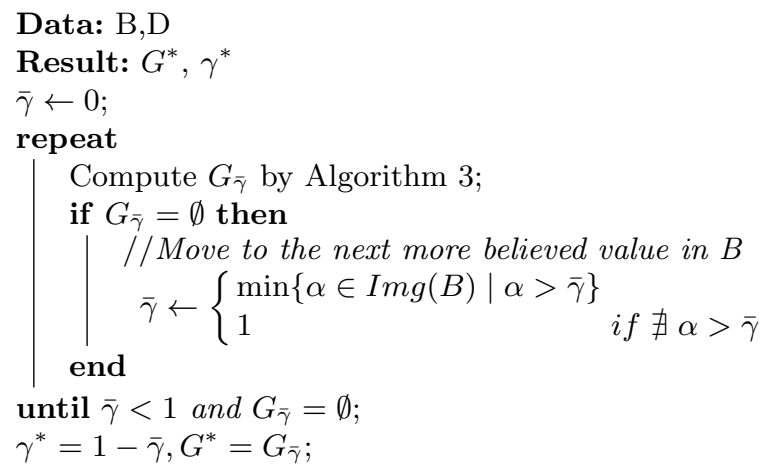

Algorithm 2: The goal election function.

where $\delta_{\psi}^{\prime}=\min \left\{\delta_{\psi}, \tau\right\}$. Desire-generation rules can be expressed by the following rule:

$$
\begin{gathered}
(5) \vDash B C: \min \left\{B\left(\phi_{1}\right) \wedge \ldots \wedge B\left(\phi_{n}\right)\right\}=\beta, D C: \\
\left.\min \left\{D^{+}\left(\psi_{1}\right) \wedge \ldots \wedge D^{+}\left(\psi_{n}\right)\right)\right\}=\delta \rightarrow D C: D^{+}(\Psi) \geq \min \{\beta, \delta\}
\end{gathered}
$$

Namely, if an agent has the beliefs $B\left(\phi_{1}\right) \wedge \ldots \wedge B\left(\phi_{n}\right)$ with a degree $\beta$ and it positively desires $D^{+}\left(\psi_{1}\right) \wedge \ldots \wedge D^{+}\left(\psi_{n}\right)$ to a degree $\delta$, then it positively desires $\Psi$ to a degree greater or equal to $\min \{\beta, \delta\}$. According to [18], goals are a set of desires that, besides being logically "consistent", are also maximally desirable, i.e., maximally justified and possible. This is expressed by the following bridge rule:

$$
(6) \vDash B C: B\left(a_{i}, \phi\right)=\beta_{\phi}, D C: D^{+}\left(a_{i}, \psi\right)=\delta_{\psi} \rightarrow G C: G(\chi(\phi, \psi))=\delta
$$

where $\chi(\phi, \psi)=\operatorname{ElectGoal}(\phi, \psi)$, as specified in Algorithm 2, is a function that allows to elect the most desirable and possible desires as goals. If ElectGoal returns $\emptyset$, then $G(\emptyset)=0$, i.e., no goal is elected.

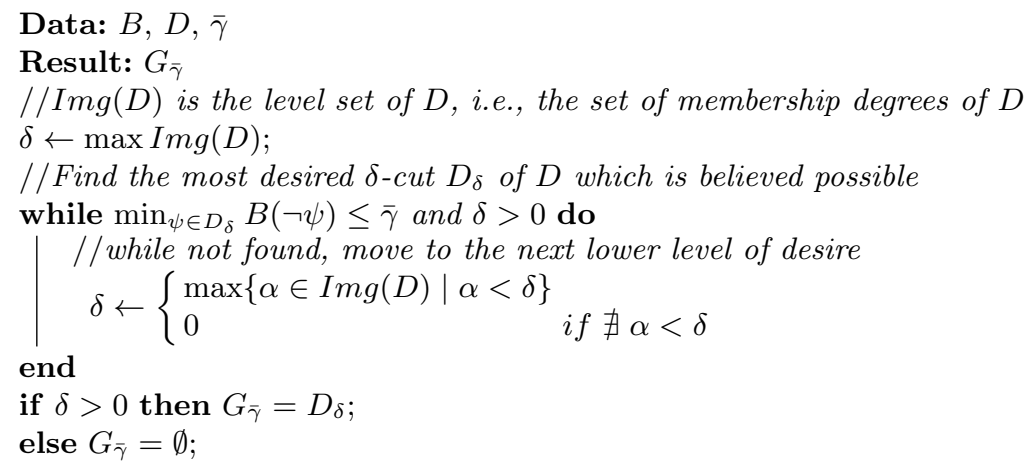

Algorithm 3: Computation of $G_{\bar{\gamma}}$. 
As expressed by the bridge rule above, once goals are generated, our agent will look for plans satisfying goal $\phi$ by applying the RequestForPlan function and do the first action of the recommended plan.

$$
\begin{gathered}
(7) \vDash G C: G\left(a_{i}, \phi\right)=\delta, P C: \text { RequestForPlan }(\phi) \rightarrow I C: \\
I\left(\text { act }_{i}, \text { PostConditon }\left(\operatorname{act}_{i}\right)\right)
\end{gathered}
$$

where RequestForPlan is a function that looks for plans satisfying goal $\phi$ in the plan library, as specified in Algorithm 1. Rule (8) means that if an agent has the intention of doing an action $a c t_{i}$ with PostCondition $\left(a c t_{i}\right)$ then it passes this information to the communication unit and via it to other agents and to the user.

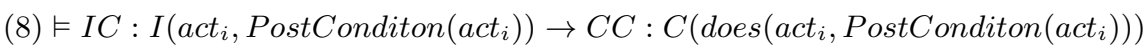

If the communication unit obtains some information that some action has been completed then the agent adds it to its beliefs set using rule $(3)$ with $B\left(\right.$ PostConditon act $\left.\left._{i}\right)\right)=$ 1.

\section{Illustrative Example}

To illustrate the reasoning process of our BDI architecture, we use our Bob's running example. To implement such a scenario using the BDI formalism, a recommender agent has a knowledge base (KB) like the one shown in Table 1, initially specified by Bob.

\begin{tabular}{ll}
\hline Beliefs & Desires \\
\hline$B(p a \rightarrow r d)=0.75$ & $D^{+}(p a)=0.8$ \\
$B(w r \rightarrow r d)=0.8$ & $D^{+}(w r)=0.8$ \\
$B(\neg e h)=0.4$ & $D^{+}(e h)=0.9$ \\
$B(b s)=0.9$ & $D^{+}(w)=0.75$ \\
\hline Table 1: Initial knowledge base of Bob's recommender agent
\end{tabular}

The belief set is represented by formulae describing the world (e.g., $B\left(\psi_{1}\right)=1$ means that $\psi_{1}$ is necessary and totally possible). Desires are all possible states that the agent wishes to achieve. Notice that they can be conflicting like $D^{+}(w r)$ and $D^{+}(\neg e h) . D^{+}(w r)=0.8$ means that $w r$ is desired to a degree equal to 0.8 . Desire-generation rules from bridge rule (5) can be described as follows:

$$
\begin{gathered}
R_{5_{1}}: B C: B(p a \rightarrow r d), D C: D^{+}(r d) \rightarrow D C: D^{+}(p a), \\
R_{5_{2}}: B C: B(w r \rightarrow r d), D C: D^{+}(r d) \rightarrow D C: D^{+}(w r), \\
R_{5_{3}}: \quad B C: B(b s), D C: D^{+}(p a) \rightarrow D C: D^{+}(w), \\
R_{5_{4}}: B C: B(p a \rightarrow w r), D C: D^{+}(w r) \rightarrow D C: D^{+}(\neg e h) .
\end{gathered}
$$

Then, the desire base of Bob, derived from desire-generation rules, will be as follows: 


$$
D=\{(p a, 0.8),(w r, 0.8),(w, 0.75),(\neg e h, 0.9)\}
$$

We may now apply rule (6) to elect Bob's goals, given his belief base and his desire base. This rule will apply the function electGoal() which will choose from the desire base the most desirable and possible desires. Then, $\operatorname{Img}(B)=$ $\{0.75,0.8,0.9,0.4\}$ and $\operatorname{Img}(D)=\{0.75,0.8,0.9\}$. We begin by calling Algorithm 2 with $\gamma=0 ; \delta$ is set to $\max \operatorname{Img}(D)=0.9$ and the corresponding desire in $D$ is $D_{\delta}=\{\neg e h\}$. Now if we verify $B(\neg(\neg e h))=0.4>\gamma$ we move to the next less desired value which sets $\delta$ to $\operatorname{Img}(D)=0.8<\delta=0.9$. $\delta=0.8>0$, then we go back to Step 2. In this case, $D_{\delta}=\{(p a, w r\}$. Now $B(\neg p a)=B(p a)=0$ because we ignore yet whether $p a$ is possible or nor. Similarly, $B(\neg w r)=0$ and Algorithm 2 will terminate with $G^{*}=G_{\gamma}=\{p a, w r\}$, i.e., Bob's recommender agent will elect as goal 'get back to a regular physical activity and reduce weight'.

Given these goals, Bob's agent $\left(a_{1}\right)$ will look in the plan library for a plan satisfying them. As explained in rule (7), the agent will invoke function RequestForPlan, which will look for a plan satisfying $p a$ and wr. Applying Algorithm 1, we have $G^{\prime}=\{p a, w r\}$ and $S^{\prime}=[p a, w r,\{p a, w r\}]$ with the same satisfaction degree $\alpha_{1}=\alpha_{2}=\alpha_{3}=0.8$. Suppose that it returns three plans $p_{1}, p_{2}$ and $p_{3}$ satisfying respectively goals $p a, w r$ and $\{p a, w r\}$. Bob's recommender agent will propose plan $p_{3}$ to the user because it meets more Bob's requirements with the same satisfaction degree. We suppose that Bob chooses plan $p_{3}$. Therefore, the first action (activity) in plan $p_{3}$ will become the agent's intention. The intended action will be proposed to the user via the communication unit by applying rule (8). Finally, if Bob starts executing the activity, information such as speed, distance or heart rate are collected via sensors (i.e., a smart watch) and transmitted to the communication unit in order to update the agent's beliefs. The revision mechanism of beliefs is the same as in [15], defined by Equation 2. Once the activity is completed, rule (3) is triggered in order to update the belief set of Bob's agent with $B$ (postCondition $($ action 1$)=1$ ) which will permit to move to the next action in plan $\alpha$.

In order to illustrate the social influence between agents, we suppose that Bob's doctor uses our application with the same goal as Bob, i.e., to reduce his diabetes risk. Then, there is a direct link between agents $a_{1}, a_{2}$ representing Bob and Bob's doctor, respectively, with $T_{a_{1}, a_{2}}(\phi)=0.9$ where $\phi$ represents any message coming from Bob's doctor (see [20] for more details). Now that Bob is executing his plan in order to get back to a physical activity, his recommender agent receives the following information from $a_{2}: B(\neg p a)=1$ which means that Bob's doctor believes that physical activity is not possible (not recommended). This information will trigger bridge rule (3). Knowing the belief degree of $a_{2}$ about $p a$ and given the trust degree of $a_{1}$ toward $a_{2}$ about information $p a\left(T_{a_{1}, a_{2}}(p a)\right), a_{1}$ decides to update its mental state according to Equation 2, and sets the new belief to $B^{\prime}(p a)=0$ according to Equation 1. This will trigger the goal generation process, which updates the elected goals. $p a$ will be removed because $B(\neg p a)=1$. Hence, a new plan is proposed to Bob. 


\section{Simulation}

Aiming to illustrate the applicability of our model, the case study used in the previous section has been implemented and simulated in the NetLogo ${ }^{6}$ environment. Netlogo [23] is a multi-agent programming language and modelling environment for simulating complex phenomena. It stands out from other agent-based simulation platforms for its ease of use and excellent documentation. We decided to use Netlogo for this work for those reasons, but also because it has a support for the BDI architecture and the Agent Communication Language (ACL).

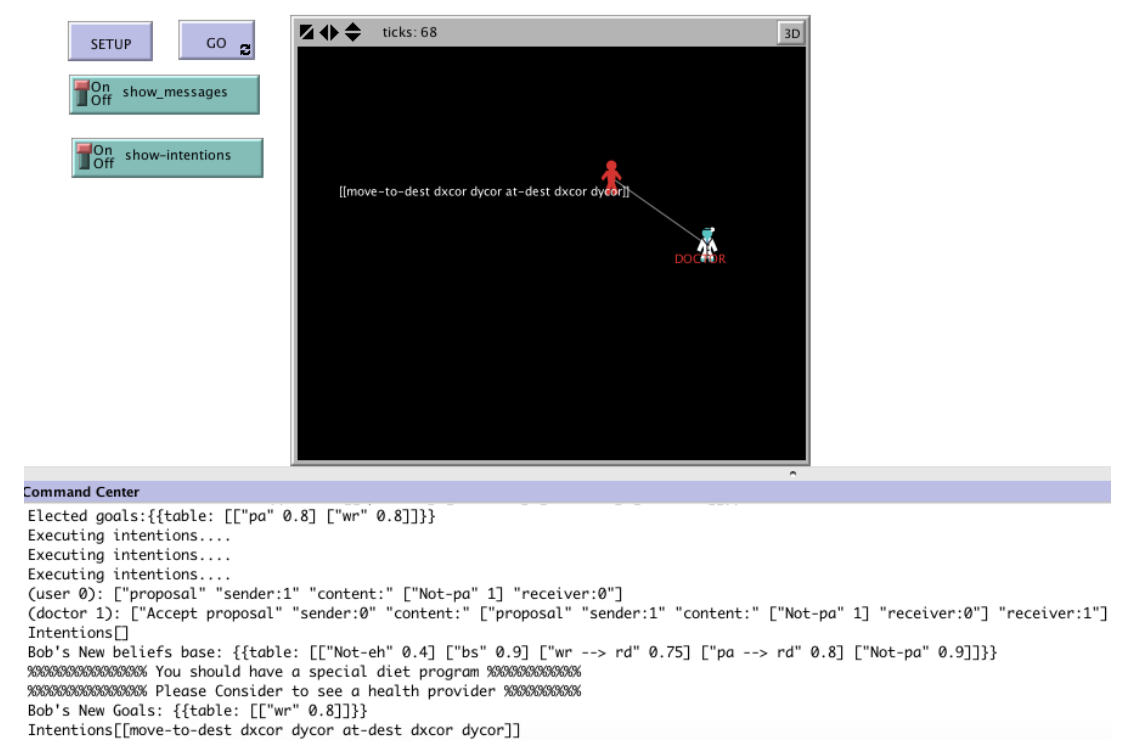

Fig. 4: A view of the simulation of our running scenario in Netlogo.

The agent-based model is composed of 2 types of agents: a user agent which represents Bob, and a doctor agent representing Bob's doctor. The behavior of Bob's agent reproduces the behavior described in Section 4 with some simplifications, e.g., in the planning context, plans are a list of activities (moving from one destination to another) defined using Netlogo procedures. The behavior of the doctor agent is similar to Bob's agent one, but in this scenario its role is limited in communicating its recommendation about physical activity to Bob's agent. Beliefs and desires are initialized according to Table 1 for Bob's agent. For the doctor agent, we initialize only its beliefs to $B(\neg p a)=1$. Figure 4 shows a general view of the implemented agent-based scenario.

As expected, in this simulation agents behave as described in the previous Section. Once the recommendation is calculated, Bob's agent starts to execute

\footnotetext{
$\overline{{ }^{6} \text { https://ccl.northwestern.edu/netlogo/ }}$
} 
its intentions. It changes its plan when it receives information from its doctor's agent, who is considered as trustworthy. The analysis of the behavior of agents in this scenario allowed to support the design of the agent-based framework, tuning and refining its specification. The simulation is available online at the following link: http://modelingcommons .org/browse/one \_model/4602.

\section{Conclusions}

Using cognitive agents architectures in the recommendation process is relevant especially in real-world applications [6]. To this end, we have presented a recommender system based on the BDI architecture. We used multi-context systems to define the BDI agent architecture with additional reasoning capabilities. First, we extended the traditional BDI architecture with a social context in which similar agents can communicate and influence each other to adopt mental attitudes. Second, we used ontologies to represent and reason about plans and intentions which offer the possibility of sharable and reusable data. The use of ontologies allow also to query streaming data. Unlike current approaches to agents recommendations, the agents (i.e, users) in our approach are active participants in the recommendation process, as they involve their personal social network based on their own perception of similarity. When applying the proposed framework to a running example, we showed it to be applicable and appropriate. As for future work, a more sophisticated evaluation of the framework with different metrics and agent strategies will be provided. As long term goals, a proof-of-concept implementation of the proposed framework is conceivable following approaches like [24].

Finally, our BDI agents operate in a dynamic environment which is dynamic both on the temporal and on the space side. Consequently, they are sensitive to the context and its changes especially spatio-temporal ones. Extending the proposed framework in order to handle spatio-temporal reasoning is then necessary. For that aim, approaches such as [25] will be explored.

\section{References}

1. Koster, A., Schorlemmer, M., Sabater-Mir, J.: Opening the black box of trust: reasoning about trust models in a bdi agent. Journal of Logic and Computation (2012) exs003

2. Pinyol, I., Sabater-Mir, J., Dellunde, P., Paolucci, M.: Reputation-based decisions for logic-based cognitive agents. Autonomous Agents and Multi-Agent Systems 24(1) (2012) 175-216

3. Montaner, M., López, B., De La Rosa, J.L.: A taxonomy of recommender agents on the internet. Artificial intelligence review 19(4) (2003) 285-330

4. Casali, A., Godo, L., Sierra, C.: Modeling travel assistant agents: a graded bdi approach. In: Artificial Intelligence in Theory and Practice. Springer (2006) 415424

5. Casali, A., Godo, L., Sierra, C.: A tourism recommender agent: from theory to practice. Inteligencia artificial: Revista Iberoamericana de Inteligencia Artificial 12(40) (2008) 23-38 
6. Casali, A., Godo, L., Sierra, C.: Validation and experimentation of a tourism recommender agent based on a graded bdi model. In: CCIA. (2008) 41-50

7. Casali, A., Godo, L., Sierra, C.: A graded bdi agent model to represent and reason about preferences. Artificial Intelligence 175(7) (2011) 1468-1478

8. Sabater-Mir, J., Cuadros, J., Garcia, P.: Towards a framework that allows using a cognitive architecture to personalize recommendations in e-commerce. In: EUMAS. (2013) 3-17

9. Bajo, J., De Luis, A., Gonzalez, A., Saavedra, A., Corchado, J.M.: A shopping mall multiagent system: Ambient intelligence in practice. In: 2nd International Workshop on Ubiquitous Computing \& Ambient Intelligence. (2006) 115-125

10. Vashisth, P., Bedi, P.: Interest-based personalized recommender system. In: Information and Communication Technologies (WICT), 2011 World Congress on, IEEE (2011) 245-250

11. Cohen, P.R., Levesque, H.J.: Intention is choice with commitment. Artificial intelligence 42(2) (1990) 213-261

12. Rao, A.S., Georgeff, M.P., et al.: Bdi agents: From theory to practice. In: ICMAS. Volume 95. (1995) 312-319

13. Wooldridge, M., Jennings, N.R., Kinny, D.: The gaia methodology for agentoriented analysis and design. Autonomous Agents and multi-agent systems 3(3) (2000) 285-312

14. Singh, M.P.: Semantical considerations on intention dynamics for bdi agents. Journal of Experimental \& Theoretical Artificial Intelligence 10(4) (1998) 551-564

15. da Costa Pereira, C., Tettamanzi, A.G.: An integrated possibilistic framework for goal generation in cognitive agents. In: Proceedings of the 9th International Conference on Autonomous Agents and Multiagent Systems: volume 1-Volume 1. (2010) 1239-1246

16. Negoita, C., Zadeh, L., Zimmermann, H.: Fuzzy sets as a basis for a theory of possibility. Fuzzy sets and systems 1 (1978) 3-28

17. Parsons, S., Jennings, N.R., Sabater, J., Sierra, C.: Agent specification using multi-context systems. In: Foundations and Applications of Multi-Agent Systems. Springer (2002) 205-226

18. da Costa Pereira, C., Tettamanzi, A.G.: Syntactic possibilistic goal generation. In: ECAI 2014-21st European Conference on Artificial Intelligence. Volume 263., IOS Press (2014) 711-716

19. Dubois, D., Prade, H.: Possibility theory and its applications: a retrospective and prospective view. Springer (2006)

20. Paglieri, F., Castelfranchi, C., da Costa Pereira, C., Falcone, R., Tettamanzi, A., Villata, S.: Trusting the messenger because of the message: feedback dynamics from information quality to source evaluation. Computational and Mathematical Organization Theory 20(2) (2014) 176-194

21. Batet, M., Moreno, A., Sánchez, D., Isern, D., Valls, A.: Turist@: Agent-based personalised recommendation of tourist activities. Expert Systems with Applications 39(8) (2012) 7319-7329

22. Gruber, T.: Ontology. Encyclopedia of database systems (2009) 1963-1965

23. Tisue, S., Wilensky, U.: Netlogo: A simple environment for modeling complexity. In: International conference on complex systems. Volume 21., Boston, MA (2004)

24. Bergenti, F., Caire, G., Gotta, D.: Agents on the move: Jade for android devices. In: Procs. Workshop From Objects to Agents. (2014)

25. Jonker, C.M., Terziyan, V., Treur, J.: Temporal and spatial analysis to personalise an agents dynamic belief, desire, and intention profiles. In: Cooperative Information Agents VII. Springer (2003) 298-315 